\title{
ESTIMATING PIG WEIGHT FROM 2D IMAGES
}

\author{
Yan Yang, Guanghui Teng* \\ Department of Agricultural and Bioenvironmental Engineering, China Agricultural \\ University, P.O. Box 195, Beijing, P. R. China \\ * Corresponding author, Address: Department of Agricultural and Bioenvironmental \\ Engineering, China Agricultural University, P.O. Box 195, Beijing, P. R. China, phone: \\ 86-10-62737583, Email: futong@cau.edu.cn
}

\begin{abstract}
This paper presents a new method that utilizes the technologies of image processing and computer vision. Firstly, the projected areas of the pig's image captured directly from top view are computed. Secondly, the heights are obtained from side view. Then the pig's weight is estimated by the projected areas and heights. By comparing with the real weight, the mean relative error is $3.2 \%$. The experiment indicates that this hands-off method has great significance in scientific management of the pig's production which does not require large labor and material resources, and also avoid the loss in production resulted from stress.
\end{abstract}

Keywords: pig; weight; image processing; projected area; height

\section{INTRODUCTION}

This paper estimates the weight of pigs from images obtained by two cameras which are installed on the top of the ceiling and side of wall. We can measure the projected areas and heights of pigs through computer vision technique (Schofield et al., 1999), and then find a close relationship between the weight and these parameters. It indicates that weights of pigs can be estimated accurately from projected areas and heights. From many experiments we obtained a regressive formulation which is used for the estimation of the pig's weight.

Yang, Y. and Teng, G., 2008, in IFIP International Federation for Information Processing, Volume 259; Computer and Computing Technologies in Agriculture, Vol. 2; Daoliang Li; (Boston: Springer), pp. 1471-1474. 


\section{IMAGE PROCESSING ALGORITHM}

\subsection{Segmentation of images}

1) Construction of the difference image. A difference image is constructed from the RGB channels, which suppresses the background and emphasizes the foreground objects (Wan et al., 2001):

$$
\mathrm{D}(\mathrm{x}, \mathrm{y})=\frac{\mathrm{g}(\mathrm{x}, \mathrm{y})+\mathrm{b}(\mathrm{x}, \mathrm{y})-\mathrm{r}(\mathrm{x}, \mathrm{y})}{[\mathrm{g}(\mathrm{x}, \mathrm{y})+\mathrm{b}(\mathrm{x}, \mathrm{y})+\mathrm{r}(\mathrm{x}, \mathrm{y})]^{1.5}}
$$

where (x,y) is the coordinate of the pixel. Figure 1(a) is an example of the difference image where the pig region is salient.

2) Automatic selection of threshold. Figure 1(b) is a typical histogram in our experiments. The pixels of the pig region concentrate on the first peak and background concentrate on the right one. (Yu et al., 2001).

3) Binarization and after-processing. A binary connected component analysis is utilized to extract a single-connectivity region. Figure $1(\mathrm{c}, \mathrm{d})$ is an example of the after-processing results.

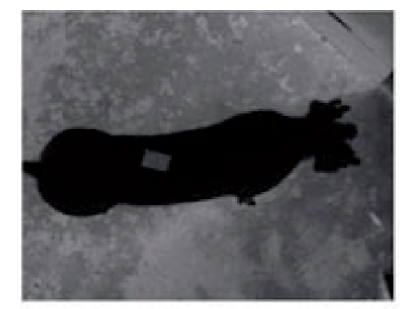

(a)

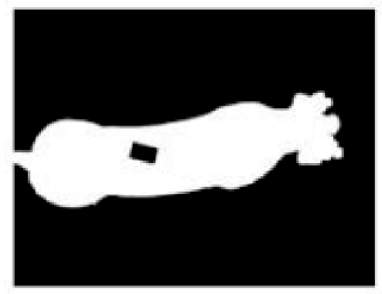

(c)

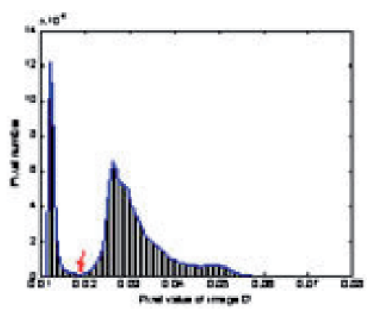

(b)

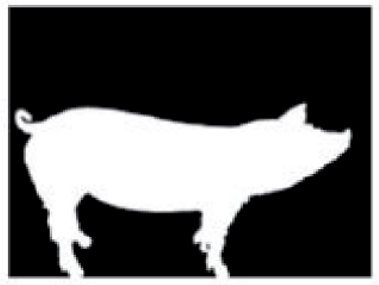

(d)

Fig. 1. Segmentation of images

(a) Difference image (b) The histogram of Difference image, threshold is 0.0262

(c) Top image after segmenting (d) Side image after segmenting 


\subsection{Measurement of projection area and height}

The calculation of projected area is to count the pixels of value 1 in the binary image, i.e. the projected area is calculated by the following equation:

$$
\frac{\mathrm{A}_{1}}{\mathrm{~N}_{1}+\mathrm{N}_{2}}=\frac{\mathrm{A}_{2}}{\mathrm{~N}_{2}}
$$

In the above equation:

A1 stands for the top projected area of pig to be calculated, $\mathrm{cm}^{2}$;

A2 stands for the real area of reference $\mathrm{cm}^{2}$;

N1 stands for the number of pixels in the segmented image of projected area after moving the reference. Pixels;

N2 stands for the number of pixels of the reference. Pixels.

We calculate the pig height from the side image. The segmented region (binary-valued pixels) of the pig is horizontally projected and the height is roughly estimated as the maximum projection value. We can obtain real height of pig by following equation:

$$
\frac{L 1}{N 1}=\frac{L 2}{N 2}
$$

L1 stands for real height of pig, $\mathrm{cm}$;

L2 stands for the real length of the reference $\mathrm{cm}$;

N1 stands for the maximum projection value as above;

$\mathrm{N} 2$ stands for the length of the reference in the segmentation image.

\section{RESULTS AND DISCUSSIONS}

As we know, volume correlates closely to weight. The pig weights were estimated using the following multiple regression equation after many experiments.

$$
\mathrm{W}=0.003 \times \mathrm{S}^{1.2811} \times \mathrm{H}^{0.6121}
$$

where $\mathrm{W}$ is the estimated weight $(\mathrm{kg})$ of a pig, $\mathrm{A}$ is specified projected image area $\left(\mathrm{cm}^{2}\right)$, and $\mathrm{H}$ is the estimated height.

On the whole, the mean relative error of this equation in these samples was $3.2 \%$. These errors partly result from the fact that the pigs did not adapt themselves to the electric balance. When driven into the balance, they did not remain stationary. Hence errors might occur when the weight is read on the display of the balance. 


\section{CONCLUSIONS AND FUTURE WORKS}

To estimate the pig weight by a hand-off method, image analysis techniques were used in this work (Minagawa et al., 1994). The results indicate that there is a strong relationship between the weight of a pig and the volume composed by projected area and height. By comparing with the real weights, the mean relative error is $3.2 \%$. More work is required to improve the image quality through carefully fixing the cameras and choosing suitable lighting, and to improve the image analysis algorithm. Measurement of weight using this method enables the stockman to monitor the performance and health of the pigs in time, and to predict and control their market weights and dates.

Future work is to develop a greater database as well as taking into account other factors, such as size and shape (Doeschl et al., 2004) which are considered as useful bases to describe the pig growth, body form and function, and also as possible indicators of muscle volume and lean content, and environmental parameters for industrial purpose. The final goal is to develop a pig management system based on the actual performance of the growing pigs. An automatic image processing system has to be developed, and images should be taken within the pens without moving pigs to special booths. This research will increase the efficiency of pig's production using the pig growth monitoring system, thereby improving pig performance without reducing the pig welfare.

\section{REFERENCES}

Schofield, C.P., J.A. Marchant et al., 1999. Monitoring pig growth using a prototype imaging system. Journal of Agric. Engineering. 72, 205-210.

Doeschl, A.B., Green, D.M., Whittemore, C.T., Schofield, C.P., Fisher, A.V. and Knap, P.W. 2004. The relationship between the body shape of living pigs and their carcass morphology and composition. Animal Science. 79: 73-83.

Minagawa, H. and T. Ichikawa. Determining the weight of pigs with image analysis. Trans. ASAE. 37(3):1011-1015.

Wan Q.Q., 1994. Digital image processing, Beijing: electronic industry publish company, 2001.

Yu X.W., Shen Z.R.. 2001, Research of insects' digital image segmentation. Transactions of the Chinese Society of Agricultural Engineering, Vol. 17(3): 137-141. 